\title{
Initial Data Sets of Minimal Energy
}

\author{
Oscar Reula \\ Max-Planck-Institut für Astrophysik, Karl-Schwarzschild-Strasse 1, \\ D-8046 Garching bei München, Federal Republic of Germany
}

\begin{abstract}
The total mass of a gravitational system, the ADM mass, is considered as a functional of the initial data sets for sources and gravitation. From the condition that such a functional has a minimum under a restricted class of variations, tensorial equations for the gravitational initial data are obtained. The solutions to these equations, whose existence for a large class of source fields is asserted, represent initial data sets for gravitation satisfying the constraint equations and may be considered as having no gravitational radiation.
\end{abstract}

\section{Introduction}

An important problem in general relativity is to compute the gravitational radiation output of isolated systems. In principle this can be done by prescribing in a hypersurface $S$ initial data for the source and the gravitational fields, evolve them according to Einstein and source field equations, and finally evaluate the News function at the $\mathscr{I}^{+}$thus generated. Besides the usual problems arising from evolving for an infinite time non-linear equations, the above program suffers from another one, namely of determining which are the appropriate data to be given at $S$ in order that the radiation we register at $\mathscr{I}^{+}$is the one generated by the source motion, for it is intuitively clear that, given any compact source field initial data one can find different initial data sets for the gravitational field such that:

i) They are consistent with the source data, in the sense that the constraint equations are satisfied, ii) the resulting source evolution is almost identical during some finite interval, but iii) the amount of radiation produced at $\mathscr{I}^{+}$is radically different.

Due to the interaction between the source and the gravitational fields the concept of the radiation produced by the source is ambiguous. Thus, there exist several procedures determining different initial data sets which pretend to capture this concept [1]. Some intend to compute in successive approximations a solution to the whole space-time and so, in pushing the problem to $\mathscr{I}^{-}$, where at least we 
have a good candidate, namely prescribing zero null datum for gravity there, but this has not yet been fully achieved. Even if it were, there would still remain some ambiguities because of the singularity at $I^{-}$, if massive field sources are considered. Others consist in taking a kind of Newtonian limit, and thus, again in successive approximations, obtaining non-radiative data.

Here we propose yet another procedure which is purely local, in the sense that one has to solve equations at some initial hypersurface, and so it is not sensitive to the details of the past source evolution, and which is purely relativistic, in the sense that it does not involve any limit of the theory. This procedure consists of minimizing the total energy, i.e. the ADM energy, keeping the sources, in a certain sense, fixed.

The idea is simple: since the ADM energy is positive when sources satisfy a local energy condition we expect that, at least for small enough fixed source configurations, there should be gravitational data compatible with the sources and minimizing the total energy. But then this data should not have any extra gravitational radiation, for if that were the case, and assuming that extra radiation contributes positively to the energy, there would be another data with lower energy. This intuitive argument is reinforced by the fact that if the sources are taken to be stationary then the minimizing energy data generates a stationary, radiation free space-time, and also by the results of a similar procedure in electromagnetism and in linearized gravity given below.

In Sect. II we give, as an example, the procedure for defining minimal energy initial data sets (MEIDS) for electromagnetism and in Sect. III for linearized gravity. In these sections we handle the equations in great detail. This is important for realizing later what happens in the full theory, treated in Sect. IV, where the equations are too cumbersome to allow for a simple description of their properties.

\section{MEIDS for Electromagnetism}

For definiteness we shall treat electromagnetism coupled minimally to a charged Klein-Gordon field $\psi$ in Minkowski space-time. Equally well we could have taken electromagnetism coupled to any other field theory which admits a Lagrangian and Hamiltonian formulation. Following [2], and choosing a flat foliation we find the energy of the system as a functional on phase space to be,

$$
E\left(\mathbf{A}, \boldsymbol{\Pi}, \psi, P_{\psi}\right)=\frac{1}{2} \int_{\mathbb{R}^{3}}\left\{\left|P_{\psi}\right|^{2}+|\mathscr{D} \psi|^{2}+m^{2}|\psi|^{2}+|\boldsymbol{\Pi}|^{2}+|\boldsymbol{\nabla} \times \mathbf{A}|^{2}\right\} d v,
$$

where $\boldsymbol{\Pi}:=-\mathbf{E}$ is the momentum conjugate to $\mathbf{A}$, the usual 3 vector potential, $P_{\psi}:=\dot{\bar{\psi}}+i e v \bar{\psi}$ the momentum conjugate to $\psi$ (with $v$ being the usual scalar potential), and $\mathscr{D} \psi=\nabla \psi+i e \mathbf{A} \psi$. We shall take as phase-space for electromagnetism $P_{\mathrm{EM}}=\left\{(\mathbf{A}, \boldsymbol{\Pi}) \in H_{\sigma+1,-1} \times H_{\sigma, 0}\right\}$ [3] $\sigma \geqq 1$, and we shall look, for each fixed $\left(\psi, P_{\psi}\right) \in C_{0}^{\infty} \times C_{0}^{\infty}$ for a pair $(\mathbf{A}, \Pi) \in P_{\mathrm{EM}}$ which minimizes $E$. Since $E$ is differentiable, nonnegative and vanishes only for the trivial solution, it is clear that if we fix $\left(\psi, p_{\psi}\right)$ nontrivially, $E$ will have a positive infimum $E\left(\psi, p_{\psi}\right)$ on $P_{\mathrm{EM}}$.

There are two problems with the program above. First since electromagnetism is a constrained theory, we have

$$
\nabla \cdot \boldsymbol{\Pi}-\frac{i e}{2}-\left(\psi P_{\psi}-\bar{\psi} P_{\bar{\psi}}\right):=\boldsymbol{\nabla} \cdot \boldsymbol{\Pi}+\varrho=0,
$$


and so we must look for a minimum of the energy in the constrained phase-space, $P_{c}$, that is, that subspace of $P_{\mathrm{EM}}$ which is compatible with (2). We shall take this into account by adding to the energy functional a term $v(\boldsymbol{\nabla} \cdot \boldsymbol{\Pi}+\varrho)$, a Lagrange multiplier, and look for a minimum of this new functional

$$
H=E+\int v(\boldsymbol{\nabla} \cdot \boldsymbol{\Pi}+\varrho) d v,
$$

which is just the Hamiltonian of the theory.

The second problem has to do with the meaning of "keeping fixed $\left(\psi, P_{\psi}\right)$ while varying $(\mathbf{A}, \boldsymbol{\Pi})$ ", since $\psi$ and $P_{\psi}$ are gauge dependent quantities. There are several ways to take this into account, they give equivalent equations. Perhaps the most direct one, shown to me by J. Ehlers, is to note that since the Hamiltonian is gauge invariant one can introduce representative fields by imposing a particular gauge condition, consider only radiations respecting that gauge, but nevertheless obtain gauge independent equations. We follow this way by imposing the Couloumb gauge, $\boldsymbol{\nabla} \cdot \mathbf{A}=0$, interpreting now $\psi$ and $\varrho_{\psi}$ as fixed complex functions and adding to the Hamiltonian a term $L(A): \int \alpha \boldsymbol{\nabla} \cdot \mathbf{A} d v, \alpha \in H_{\sigma+2,-2}$, a Lagrange multiplier. A necessary condition then for the existence of an energy minimum is that the differential of $H(\mathbf{A}, \pi)+L(\mathbf{A})$ vanishes, that is

$$
\begin{gathered}
D H_{(\mathbf{A}, \mathbf{\Pi})}(\delta \mathbf{A}, \delta \mathbf{\Pi})+D L_{(\mathbf{A}, \mathbf{\Pi})}(S \mathbf{A})=0, \\
\forall(\delta \mathbf{A}, \delta \mathbf{\Pi}) \in T_{(\mathbf{A}(\mathbf{\Pi})} P_{\mathrm{EM}} .
\end{gathered}
$$

Using Hamiltonian's equations and identifying the linear map $D H$ with its $L_{2}$ representative we have,

$$
\begin{gathered}
\dot{\mathbf{A}}:=-\frac{\delta H}{\delta \Pi}=-\boldsymbol{\Pi}+\nabla v, \\
\dot{\boldsymbol{\Pi}}:=\frac{\delta H}{\delta A}=-\nabla \times \nabla \times \mathbf{A}+\mathbf{J},
\end{gathered}
$$

where $\mathbf{J}=\frac{i e}{2}(\psi \mathscr{D} \bar{\psi}-\bar{\psi} \mathscr{D} \psi)$, and (4) becomes,

$$
\begin{gathered}
\boldsymbol{\Pi}-\boldsymbol{\nabla} V=0, \\
\boldsymbol{\nabla} \times \boldsymbol{\nabla} \times \mathbf{A}-\mathbf{J}=\boldsymbol{\nabla} \alpha \quad \text { for some } \alpha \in H_{\sigma+2,-2} .
\end{gathered}
$$

Note that if we now relax the gauge condition and perform a gauge transformation, then Eqs. (6-7) are gauge invariant.

Physically one would like to be able to specify instead of $\psi$ and $p_{\psi}$ the gauge invariant quantities $\varrho$, and $\mathbf{J}$. Unfortunately this is not possible for $\mathbf{J}$ does not only depend on $\psi$, but also on the connection $\mathbf{A}$, and so it can only be determined a posteriori, i.e. when Eqs. (6-7) are solved. To obtain solutions to the above system, Eqs. (2,6-7), we must again impose gauge conditions, take fixed representatives $\psi, p_{\psi}$, solve for unique $(\mathbf{A}, \boldsymbol{\Pi})$ and so obtain an array $\left\{\left(\psi, p_{\psi}\right),(\mathbf{A}, \boldsymbol{\Pi})\right\}$, (determined up to gauge transformations) of initial data. It is only from this point on that a physical description of the system makes sense.

Another way to take into account the fixing of the sources is to use a global splitting of the tangent phase space into $L^{2}$ orthogonal subspaces corresponding to 
radiative, longitudinal and pure gauge modes, and to require that $H$ has a minimum only along radiative and longitudinal directions, that is $d H \in$ gauge subspace. We follow this way using a splitting of $T P_{\mathrm{EM}}$ analogous to the one introduced for gravity in [4]. Since in this splitting the gauge space is just

$$
\left[\begin{array}{rr}
0 & -1 \\
1 & 0
\end{array}\right]\left(D \phi_{(\mathbf{A}, \mathbf{\Pi})}\right) *\left(H_{\sigma+2,-2}\right)=\left\{(\nabla \alpha, 0), \alpha \in H_{\sigma+2,-2}\right\}
$$

that is the image of $H_{\sigma+2,-2}$ under the $L^{2}$ dual of the differential of the constraint map

$$
\Phi(\mathbf{A}, \boldsymbol{\Pi})=\left(\begin{array}{c}
0 \\
\nabla \cdot \mathbf{\Pi}
\end{array}\right)
$$

we obtain again Eqs. (6) and 7.

A similar splitting holds also for gravitation [4].

We look now for a solution to system (6), (7). Consider first (6). Using the constraint equation (2) we get,

$$
\Delta V=-\varrho,
$$

which has a unique solution $v \in H_{\sigma+1,-1}$ and so $\Pi=\nabla V \in H_{\sigma, 0}$.

We consider now Eq. (7). It is easy to see that this equation has an integrability condition. Taking its divergence we obtain

$$
-\nabla \cdot \mathbf{J}=\Delta \alpha .
$$

which we take as an equation determining $\alpha$.

Since for the gravitational case the integrability conditions are not so easy to derive it is of interest to see how we can arrive at (10) from a general principle. Since the constraint is preserved under time evolution we should have,

$$
D \Phi(\dot{\mathbf{A}}, \dot{\mathbf{\Pi}})=(0, \dot{\varrho})=(0,-\nabla \cdot \mathbf{J}),
$$

where we have used the conservation equation which the sources must satisfy if the Lagrangian is to be gauge invariant. Since $\dot{\mathbf{I}}=\nabla \alpha$ we see that (11) is just (10), and so the integrability condition can be obtained by applying $D \Phi$ to both sides of (6), (7) considered as a pair. We will follow an identical procedure in the gravitational case.

To obtain a unique A we fix again its gauge freedom in the usual way, namely requiring that $\boldsymbol{\nabla} \cdot \mathbf{A}=0$. Thus we obtain the coupled elliptic system,

$$
\begin{gathered}
\Delta \mathbf{A}+(\nabla \alpha+\mathbf{J})=0, \\
\Delta \alpha+\nabla \cdot \mathbf{J}=0 .
\end{gathered}
$$

In our case $\mathbf{J}$ is linear in $\mathbf{A}$, and the dependence is such that one can show that given $\left(\psi, P_{\psi}\right) \in C_{0}^{\infty}$ there is a unique pair $\mathbf{A}, \alpha$ with $\mathbf{A} \in H_{\sigma+1,-1}^{\cdot}, \boldsymbol{\nabla} \cdot \mathbf{A}=0$, and $\alpha \in H_{\sigma,-2}$, solving $(12 \mathrm{a}, \mathrm{b})$.

In the general case, when $\varrho$ and $\mathbf{J}$ depend smoothly on $(\mathbf{A}, \boldsymbol{\Pi})$ but in a non-linear way one can only assert, using the implicit function theorem, the existence of a pair $(\mathbf{A}, \boldsymbol{\Pi})$ satisfying the now coupled system $(9),(12 a, b)$, for sources in a small neighborhood of zero. 
Note that since the energy functional is positive definite and the pair $(\mathbf{A}, \boldsymbol{\Pi})$ satisfying (4) is unique (up to gauge transformations) it must be the minimum we are looking for.

\section{MEIDS for Linearized Gravity}

We consider a Hamiltonian formalism for linearized gravity around a flat metric and with respect to a flat foliation. We do not take any particular source, we only require that these sources also admit a Hamiltonian formulation for their equations of motion. As phase space for linearized gravitation we take the space of pairs $\left(h_{a b}, \omega^{a b}\right) \in H_{\sigma+1,-1}^{\cdot *} \times H_{\sigma, 0}^{\cdot *} \sigma \geqq 1$ of linearized 3 -metrics and their conjugate momenta [5].

Using a similar argument to the second one used in the preceding section we obtain a necessary condition for the existence of a minimum, namely.

$$
\left(-\dot{\omega}^{a b}, \dot{h}_{c d}\right) \in\{\text { gauge space }\} \text {. }
$$

where as before $\left(-\dot{\omega}^{a b}, \dot{h}_{c d}\right)$ is obtained using Hamilton's equations. The same result, namely Eqs. $(16 \mathrm{a}-\mathrm{b})$ can be obtained using the alternative way of fixing sources and imposing the following gauge conditions:

$D_{a}\left(a^{a b}-\frac{1}{2} l^{a b} h\right)=\left(\Delta \omega-\nabla_{a} \nabla_{b} \omega^{a b}\right)=0$ as Lagrange multipliers.

$$
\begin{gathered}
\dot{h}_{a b}:=\frac{-\delta H}{\delta \omega^{a b}}=-2\left(\omega^{a b}-\frac{\omega}{2} e^{a b}\right)+2 D^{(a} \beta^{b)}, \\
\dot{\omega}^{a b}:=\frac{\delta H}{\delta h_{a b}}=e^{a b} D^{2} \alpha-D^{a} D^{b} \alpha+G_{L}(h)^{a b}-S^{a b},
\end{gathered}
$$

where $e_{a b}$ is the flat metric about which we are linearizing (all indices are raised or lowered with respect to it), $D_{c}$ its covariant derivative, and

$$
G_{L}(h)^{a b}=-\frac{1}{2} D^{2} h^{a b}-\frac{1}{2} D^{a} D^{b} h+\frac{1}{2} e^{a b}\left(D^{2} h-D_{c} D_{d} h^{c d}\right)+D^{(a} D_{c} h^{b) c} .
$$

its linearized Einstein tensor, and $\left(\alpha, \beta^{a}\right)$ are the Lagrange multipliers corresponding to $v$ in electromagnetism, here $1+\alpha$ and $\beta^{a}$ are interpreted as lapse and shift respectively. The tensor $S^{a b}$ is the space-space projection of the energy momentum tensor of the sources fields.

As in electromagnetism we can characterize the gauge space as

$$
\left(\begin{array}{rr}
0 & -1 \\
1 & 0
\end{array}\right)\left(D \phi^{*}\right)\left(H_{\sigma+2,-2}, H_{\sigma+2,-2}^{\cdot}\right)
$$

That is, since

$$
\Phi(h, \omega)=\left(\begin{array}{c}
-D^{2} h+D_{c} D_{d} h^{c d} \\
2 D_{a} \omega^{a b}
\end{array}\right),
$$

as the set of all pairs $\left(2 D_{(a} X_{b)},-e^{a b} D^{2} C+D^{a} D^{b} C\right)$ with

$$
\left(C, X^{b}\right) \in H_{\sigma+2,-2} \times H_{\sigma+2,-2}^{\cdot} \text {. }
$$


Thus, from (13), (14a, b), and (15) we obtain

$$
\begin{gathered}
-2\left(\omega^{a b}-\frac{\omega}{2} e^{a b}\right)+2 D^{(a} \beta^{b)}=-e^{a b} D^{2} C+D^{a} D^{b} C, \\
+e^{a b} D^{2} \alpha-D^{a} D^{b} \alpha+G_{L}^{a b}-S^{a b}=2 D^{(a} X^{b)} .
\end{gathered}
$$

Note the approximate symmetry between (16a) and (16b) under interchange of $\left(\alpha, \beta^{a}\right)$ and $\left(C, X^{a}\right)$. This symmetry, which is much more apparent in the full gravitational case, is due to the fact that in the $3+1$ decomposition the gauge transformations are realized by integrating Eqs. (14a) and (14b) with different lapse-shift pairs.

The above Eqs. $(16 \mathrm{a}, \mathrm{b})$ together with the constraint equations,

$$
\Phi(h, \omega)=\left(\begin{array}{c}
2 \varrho \\
2 J^{a}
\end{array}\right),
$$

where $\varrho$ and $J^{a}$ are the time-time component and the time-space projection of the energy-momentum tensor respectively, determine unique (up to gauge transformations) pairs $\left(h_{a b}, \omega^{c d}\right)$ and $\left(\alpha, \beta^{a}\right)$ satisfying them. As we shall see below, in the process of solving them we also determine $C$ and $X^{a}$, but not uniquely, unless a mild condition is imposed on the sources.

As in the former case we also encounter here integrability conditions (which are equations for $C$ and $\left.X^{a}\right)$. Again we find them applying to both sides of (16a) and (16b) the operator $D \Phi$. We obtain,

$$
\begin{gathered}
D^{4} C=-D_{a} J^{a}, \\
D^{2} X^{b}+D^{b} D_{a} X^{a}=-D_{a} S^{a b},
\end{gathered}
$$

a pair of elliptic equations for $C$ and $X^{b}$.

These integrability conditions can also be understood in two other ways. First note that if we could assume that the energy momentum of the sources is conserved (something which we do not assume) then from the evolution point of view we would have.

$$
D \Phi\left(\dot{g}_{a b}, \dot{\Gamma}^{c d}\right)=\left(\begin{array}{c}
2 \dot{\underline{Q}} \\
2 \dot{J}^{b}
\end{array}\right)=\left(\begin{array}{c}
-2 D_{a} J^{a} \\
-2 D_{a} S^{a b}
\end{array}\right),
$$

which are the right-hand sides of the above equations. Second note that from Eq. (16a) we have

$$
\omega^{a b}=D^{(a} \beta^{b)}-e^{a b} D_{c} \beta^{c}-\frac{1}{2}\left(D^{a} D^{b} C+e^{a b} D^{2} C\right) .
$$

Taking its divergence and using the constraint we obtain an equation for $\beta^{c}$, namely

$$
D_{a} D^{[a} \beta^{b]}=-J^{b}-D^{b} D^{2} C,
$$

from which the integrability condition is apparent. Taking its divergence we obtain.

$$
0=D_{b} J^{b}+D^{4} C
$$


which is just (18a). Note that, once $C$ is known, Eq. (20) determines $\beta^{a}$ only up to a gradient, $D^{a} \Phi$, and so Eq. (19) determines $\omega^{a b}$ only up to its gauge freedom $\omega^{a b} \rightarrow \omega^{a b}+e^{a b} D^{2} \Phi-D^{a} D^{b} \Phi$. Similarly, Eq. (18b) can be seen to be a consequence of the linearized Bianchi identities, $D_{a} G_{L}^{a b}=0$. Thus we have obtained a set of Eqs. $(16 \mathrm{a}, \mathrm{b}),(17)$, and $(18 \mathrm{a}, \mathrm{b})$ which determine uniquely, up to gauge transformations, $h_{a b}, \omega^{a b}, \alpha, \beta^{a}, C, X^{a}$.

Before proceeding to solve these equations it is convenient to reexpress them in other variables. To do this we define $\Phi^{a b}=-h^{a b}+e^{a b}\left(\frac{h}{2}+\alpha\right)$. This is the analoge, in the full theory, to conformally transforming the metric to $\tilde{g}_{a b}=e^{-2 \alpha} g_{a b}$ and then defining, choosing a background flat metric $e_{a b}, \varpi^{a b}=e^{a b}-\sqrt{\tilde{g}} \tilde{g}^{a b}$. Furthermore it is convenient to fix, once for all, the gauge freedom. We do this in the usual way [7], namely requiring $D_{a} \Phi^{a b}=\omega=0$.

We thus obtain the following reduced system:

a)

b)

c)

d)

e)

$$
\begin{gathered}
e^{a b} D^{2} C-D^{a} D^{b} C+2 \omega^{a b}-2 D^{(a} \beta^{b)}=0, \\
D^{2} \Phi^{a b}-2 S^{a b}+4 D^{(a} X^{b)}=0 \\
D^{2} \alpha-\frac{1}{2}\left(S+\varrho+2 D_{c} X^{c}\right)=0 \\
D^{2} \beta^{b}+2 J^{b}=0 \\
D^{4} C+D_{a} J^{a}=0 \\
D^{2} X^{b}+D^{b} D^{c} X_{c}-D_{a} S^{a b}=0
\end{gathered}
$$

If we assume the sources to be independent of $(h, \omega)$, as is usually the case for linearized gravity in contrast to the scalar electrodynamics of the preceding sections, and that they are of compact support and belong to the following Sobolev spaces:

$$
\varrho \in H_{\sigma-1,10}, \quad J^{a} \in H_{\sigma-1,1}^{\cdot}, \quad \text { and } \quad S^{a b} \in H_{\sigma-1,1}^{\cdot} .
$$

we obtain the following theorem.

Theorem. If the above assumptions about the sources are made, then there exists a unique (up to gauge) pair $(h, \omega) \in H_{\sigma+1,-1}^{\cdot} H_{\sigma, 0}$ satisfying (16a, b) and (17).

Proof. We first show existence of a solution $\left(\Phi^{a b}, \omega^{a b}, \alpha, \beta^{a}, C, X^{a}\right)$ to Eqs. (22a-f) and then show that it satisfies $D_{a} \Phi^{a b}=\omega=0$ and so, it produces a unique $(h, \omega)$ solution to $(16 \mathrm{a}, \mathrm{b})$ and (17).

We first show existence of solutions to the decoupled Eqs. (22d-f). From the general theory of elliptic equations in Sobolev spaces [4] it is easy to show there exists a unique $\beta^{a} \in H_{\sigma+1,-1}$ solution to (22d).

Since the kernel of $D^{4}$ in $H_{\sigma+2,-2}$ is just the subspace of constant functions and the source in Eq. (22e), namely $D_{a} J^{a}$, is orthogonal in the $L^{2}$ sense to this subspace, there exists a unique (up to constants) solution $c \in H_{\sigma+2,-2}$ to (22e). Note that in general $c$ will go asymptotically as a logarithm (although $\omega^{a b}$ will still go as $1 / r^{2}$ ). This has a space-time interpretation as a boost of the hypersurface. In fact this logarithmic behaviour can be avoided by considering sources whose total 4-momentum is orthogonal to the foliation, that is whose total 3-momentum vanishes. 
The kernel of the elliptic operator in (22f) consist only of the zero element and so there exists a unique $X^{b} \in H_{\sigma,-1}$ satisfying (22f). But the source in this equation is of compact support and is a pure divergence and so $X^{b} \in H_{\sigma, 0}$.

Equation (22a) determines $\omega^{a b}$ in terms of $C$ and $\beta^{a}$. Since $C \in H_{\sigma+2,-2}$ and $\beta^{a} \in H_{\sigma+1,-1}$ we have, $\omega^{a b} \in H_{\sigma, 0}^{\cdot \cdot}$. Since $X^{b} \in H_{\sigma, 0}$ and since the Laplacian is an isomorphism between $H_{\sigma+1,-1}$ and $H_{\sigma-1,1}$ we conclude that there exist unique $\Phi^{a b} \in H_{\sigma+1,-1}^{\ddot{*}}$ and $\alpha \in H_{\sigma+1,-1}$ satisfying (22b) and (22c) respectively. Thus we have shown existence of a solution to the reduced system $(22 \mathrm{a}-\mathrm{f})$.

Taking the divergence of (22b) using (22f) and the injectivity of the Laplacian we conclude $D_{a} \Phi^{a b}=0$. On the other hand, taking the trace of (22a), and using (22d) and (22e) we conclude $\omega=0$. Thus our gauge conditions are consistent and our solution produces also a solution to $(16 \mathrm{a}, \mathrm{b})$ and (17). Again, as in the case of electromagnetism we see that our procedure sets to zero as much as possible of the time derivatives of $h_{a b}$, and $\omega^{a b}$ in the evolution equations. In particular, if the sources are compatible with a stationary solution, namely if $D_{b} J^{b}=D_{b} S^{b a}=0$, we can see that $C=$ cte, $X^{b}=0$, that is the integrability conditions are automatically satisfied, and so $\dot{h}_{a b}=\dot{\omega}^{a b}=0$. Thus the gravitational data is also compatible with a stationary solution. If furthermore we have $J^{b}=0$, then $\beta^{a}=0$ and so we also have $\omega^{a b}=0$ and the gravitational data are also compatible with a static solution. Note that we do not assert that the solution will be stationary (or static) since we do not assume any particular model for the evolution of the sources.

\section{The Gravitational Case}

As in the former cases we proceed to deduce the equations using the phase space splitting. If would be interesting to see whether there are gauge conditions which when used as Lagrange multipliers give the equations below. Varying the total Hamiltonian, $H=H\left(g_{a b}, \Pi^{a b}\right.$, matter fields) with respect to $\Pi^{a b}$ and $g_{a b}$ we obtain,

$$
\begin{gathered}
\dot{g}_{a b}:=-\frac{\delta H}{\delta \Pi^{a b}}=-2 N g^{-1 / 2}\left(\Pi_{a b}-g_{a b} \Pi / 2\right)+2 D_{(a} N_{b)}, \\
\dot{\Pi}^{a b}:=\frac{\delta H}{\delta g_{a b}}=g^{1 / 2}\left\{-D^{a} D^{b} N+g^{a b} D^{2} N+N\left(\gamma^{a b}-S^{a b}\right)\right\}+\underset{N^{c}}{\mathscr{L}} \Pi^{a b},
\end{gathered}
$$

where $\left(N, N^{a}\right)$ is a lapse-shift pair $\left(N, N^{a}\right.$ appear as Lagrange multipliers in our variation), $D_{a}$ is the covariant derivative operator associated with $g_{a b}$, and

$$
\gamma^{a b}=G^{a b}+g^{-1}\left[\left(2 \Pi^{a c} \Pi_{c}^{b}-\Pi^{a b} \Pi\right)-g_{4}^{a b}\left(2 \Pi^{c d} \Pi_{c d}-\Pi^{2}\right)\right],
$$

where $G^{a b}$ is the Einstein tensor associated to $g_{a b}$.

As before, the splitting condition is

$$
\left(\Pi^{a b}, g_{a b}\right) \in \operatorname{Range}\left(\begin{array}{cc}
0 & \sqrt{g} \\
-1 / \sqrt{g} & 0
\end{array}\right)\left(D \Phi_{(g, \Pi)}\right)^{*},
$$

which is the gauge space [6], $\Phi$ being now the constraint map of gravitation,

$$
\phi\left(g_{a b}, \Pi^{a b}\right)=\left(\begin{array}{c}
-2 \gamma \\
2 D_{a}\left(g^{-1 / 2} \Pi^{a b}\right)
\end{array}\right) .
$$


We thus arrive at the equations,

$$
\begin{aligned}
h_{a b}:= & -2 N g^{-1 / 2}\left(\Pi_{a b}-g_{a b} \frac{\Pi}{2}\right)+2 D_{(a} N_{b)}+D_{a} D_{b} C-g_{a b} D^{2} C \\
& -C \gamma_{a b}-g^{-1 / 2}\left(\begin{array}{l}
\mathscr{L} \Pi^{e d} \\
x^{e}
\end{array}\right) g_{c a} g_{d b}=0, \\
\omega^{a b}:= & g^{1 / 2}\left[-D^{a} D^{b} N+g^{a b} D^{2} N+N\left(\gamma^{a b}-S^{a b}\right)-2 D^{(a} X^{b)}\right] \\
& +2 C\left(\Pi^{a b}-g^{a b} \frac{\Pi}{2}\right)+\underset{N^{c}}{\mathscr{L} \Pi^{a b}}=0 .
\end{aligned}
$$

The above equations, together with the constraint equation,

$$
\Phi\left(g_{a b}, \Pi^{a b}\right)=\left(\begin{array}{c}
-2 \gamma \\
2 D_{a}\left(g^{-1 / 2} \Pi^{a b}\right)
\end{array}\right)=\left(\begin{array}{c}
2 \varrho \\
2 J^{b}
\end{array}\right)
$$

should determine a unique pair $\left(g_{a b}, \Pi^{c d}\right)$, solution to them and so minimizing the total mass of the system. The approximate symmetry we noted in the linearized case is now much more apparent in Eqs. (24a) and (24b). Under the interchange of $\left(N, N^{a}\right)$ with $\left(C, X^{a}\right)$ they go into one another except for the source term $S_{a b}$.

In this case we also encounter integrability conditions which again can be found by applying to the above equations the map $D \Phi_{(g, \Pi)}(h, \omega)$. They are a pair of linear elliptic equations for $C$ and $X^{a}$ of fourth and second order respectively.

All the equations are tensorial and do not depend on any metric background, so they are gauge invariant. We proceed now to study their solutions. In contrast to the linear cases, to "give the sources on the manifold" does not give rise to a unique solution, and so to a unique initial data set. The reason for that is the absence of any metric background with respect to which one could fix the sources. To see this consider the following example (R. Penrose, personal communication): Let the source be a scalar-wave field with vanishing momentum, $p_{\phi}=0$, that is a scalar function $\phi$. Further assume that $\phi$ has topologically spherical constant value surfaces, a unique maximum with value $\phi_{0}$ and compact support. Then this field cannot give rise to a unique solution with minimal energy, for if that were the case then the value of that energy should depend only on $\phi_{0}$, but since $\phi_{0}$ is dimensionless it would be zero. In fact there are many physically different solutions, one for each flat background one chooses to take [8]. We consider this non-uniqueness a physically desirable fact, for otherwise we would not have enough physical freedom to distribute the sources. To see this suppose one is interested in describing the collision of two fluid balls which start at rest some distance apart. Without a background metric this distance cannot be given a priori, and so if the solution were unique we would not be able to choose it at will.

An important, and simpler to handle, class of source configurations is that of momentarily static sources, $J_{a}=0$. For this class we can set, $\omega^{a b}=\beta^{a}=C=0$, and obtain a reduced system for $g_{a b}, X_{a}, N$, which we call the momentarily static equations. They are,

$$
\begin{gathered}
N\left(G_{a b}-S_{a b}\right)+g_{a b} D^{2} N-D_{a} D_{b} N-2 D_{(a}-X_{b)}=0 \\
2 D^{a} D_{(a} X_{b)}+\left(\varrho D_{b} N+D^{a}\left(N S_{a b}\right)\right)=0 \\
R(g)-2 \varrho=0
\end{gathered}
$$


where (26c) is the integrability condition for (26b). Note that if the source functionals, $\varrho$ (g, matter fields), $S_{a b}(g$, matter fields) are chosen in such a way that whenever (26a) and (26c) are satisfied the second term of (26b) vanishes. We then have $X^{b}=0$ and the system reduces further to the static Einstein equations.

We proceed now to establish existence of solutions to the momentarily static equations for small enough source configurations. We shall make them unique by fixing a flat background.

Theorem. Fix any flat background metric, $e_{a b}$, which we use to fix the gauge and the sources, and let $\left(\varrho, S_{a b}\right) \in H_{\sigma-1,1} \times H_{\sigma-1,1}^{\cdot}, \sigma \geqq 2$, depend smoothly on $g_{a b} \in H_{\sigma+1,-2}^{\cdot}$. Then there exist a $\varepsilon>0$ such that for $0 \leqq \lambda \leqq \varepsilon$ there exists a unique (up to diffeomorphisms) solution $\left(g_{a b}, X_{a}, N\right) \in H_{\sigma+1,-2}^{\cdot *} \times H^{\cdot} \times H_{-2}$ to the momentarily static equations when $\left(\varrho, S_{a b}\right)$ is replaced by $\left(\lambda \varrho, \lambda S_{a b}\right)$.

Proof. We follow standard methods to reduce the above system to an almost elliptic one, i.e. a system whose linearization about the trivial solution is elliptic, and then apply the implicit function theorem and the theory of linear elliptic operators to establish existence.

To render the system elliptic we first follow the usual method for the static Einstein equations and apply to the above system the following conformal transformation, $\hat{g}_{a b}=N^{2} g_{a b}$. This transformation gets rid of second derivatives of $N$ from Eq. (26a). Second we follow $[9,10]$ and define new variables $\phi^{a b}:=e^{a b}$ $-\sqrt{\hat{g}} \hat{g}^{a b}, U:=-\ln N$. Finally we require the equations $\psi^{b}:=\hat{D}_{a} \phi^{a b}=0$, where $\hat{D}_{a}$ is the covariant derivative associated to $e_{a b}$. Thus we obtain a reduced system of equations with the following form.

$$
\begin{gathered}
E^{a b}\left(\Phi^{c d}, X^{c}, U, \lambda\right):=\hat{g}^{c d} \widetilde{D}_{c} \widetilde{D}_{d} \Phi^{a b}+\text { terms } \\
E^{a}\left(\Phi^{c d}, X^{c}, U, \lambda\right):=2 \widetilde{D}_{a} \hat{g}^{a b} \widetilde{D}_{(b} X_{c)}+\text { terms } \\
E\left(\Phi^{c d}, X^{c}, U, \lambda\right):=\hat{g}^{c d} \widetilde{D}_{c} \widetilde{D}_{d} u+\text { terms }
\end{gathered}
$$

where the extra terms are such that they do not contribute to the linearized (around $\Phi^{a b}=\chi^{a}=u=\lambda=0$ ) equations. They contain only up to second derivatives of $\Phi^{a b}$ and $u$, except for $\lambda \varrho$ and $\lambda S_{a b}$.

Note that while in the original system $(26 a-c)$ Eq. $(26 b)$ is redundant, it is just the integrability condition for (26a), in the reduced system Eq. (27b) is not, and we must solve the whole system.

We next establish existence of solutions to the reduced system and then show that they are also solutions to the momentarily static system.

Lemma 1. There exist $\varepsilon^{\prime}>0$ such that for $0 \leqq \lambda \leqq \varepsilon^{\prime}$ there exist a differentiable function

$$
\psi(\lambda)=\left(\Phi^{a b}(\lambda), X^{a}(\lambda), u(\lambda)\right):\left[0, \varepsilon^{\prime}\right] \rightarrow X=H_{\sigma+1,-1}^{\cdot \cdot} \times H_{\sigma, 0}^{\cdot} \times H_{\sigma+1,-1}, \quad \sigma \leqq 2,
$$

To show this we use the implicit function theorem for Banach spaces $[1<]$. Consider the reduced system as a function from $(\psi, \lambda):=\left(\Phi^{a b}, X^{a}, U, \lambda\right) \in X \times \mathbb{R}^{+}$ into $Y=H_{\sigma-1,1}^{\cdot \bullet} \times S^{*} \times H_{\sigma-1,1}$, where $S^{\cdot}$ is the Hilbert space of vectors fields 
$l^{a} E H_{\sigma-2,2}$ of the form $l^{a}=\widetilde{D}_{b} m^{a b}, m^{a b} \in H_{\sigma-1,1}^{\ddot{\prime}}$,

$$
F(\psi, \lambda):=\left[\begin{array}{c}
E^{a b}(\psi, \lambda) \\
E^{a}(\psi, \lambda) \\
E(\psi, \lambda)
\end{array}\right] .
$$

This function is continuously differentiable if $\sigma \geqq 2$, and furthermore $F(0,0)=0$. Thus if its differential with respect to $\psi$ at $\psi=\lambda=0 . D_{(0} F_{0)}(\delta \psi)$, were an isomorphism between $X$ and $Y$, the implicit function theorem would then assert the existence of an $\varepsilon^{\prime}>0$ with the properties required on the lemma. But

$$
D F_{(0,0)}(\delta \psi)=\left[\begin{array}{c}
e^{c d} \widetilde{D}_{c} \widetilde{D}_{d} \Phi^{a b} \\
2 e^{a b} \widetilde{D}_{a} \widetilde{D}_{(b} X_{c)} \\
e^{c d} \widetilde{D}_{c} \widetilde{D}_{d} u
\end{array}\right],
$$

which from the results in [4] is an isomorphic map. We complete the proof of the theorem using the following lemma.

Lemma 2. There exist $\varepsilon, 0<\varepsilon \leqq \varepsilon^{\prime}$ such that for $0 \leqq \lambda \leqq \varepsilon$ every solution to the reduced system is also a solution to the momentarily static system.

We prove this by showing that if $\Phi^{a b}(\lambda)$ is a solution then $\psi^{b}=\tilde{D}_{a} \Phi_{(\lambda)}^{a b}=0$. Taking the divergence of Eq. (27a) and using (27b) we obtain a linear homogeneous equation for $\psi^{b} \in H_{\sigma, 0}$. This equation depends continuously on $\lambda$ and for $\lambda=0$ is the Laplacian, $e^{a b} \widetilde{D}_{a} \widetilde{D}_{b} \psi^{c}=0$. Since the Laplacian is injective in $H_{\sigma, 0}$ and since injectivity is a continuous property of elliptic operators we conclude that $\exists \varepsilon$, $0<\varepsilon \leqq \varepsilon^{\prime}$, such $\psi^{c}(\lambda)=0$.

In the case that the sources admit an equilibrium configuration, [see note below Eq. (26c)], we obtain existence of solutions to the stationary Einstein equations, which clearly do not contain radiation. Only for this case our prescription for picking initial data is equivalent to the non-incoming radiation condition of $\mathscr{I}^{-1}$.

Existence of solutions to Eqs. (24a, b) and (25) can probably be established for arbitrary but sufficiently small sources by employing a similar method to the one used above. We expect this to be the case because the linearized equations (around $g_{a b}=e_{a b}, \Pi^{a b}=0$ ), that is the system treated in the preceding section, are elliptic and have a unique (up to gauge) solution.

In Sect. III we saw that $C$ will in general have a logarithmic behaviour but also that it can be eliminated by requiring the vanishing of the total 3-momentum of the source. This can always be achieved by "boosting" the sources. Although in the linearized case this logarithmic behaviour is not important, in the non-linear one it can be disastrous. In fact, in this case $C$ couples with $g_{a b}$ in such a way that we would also have a logarithmic behaviour in $g_{a b}$. Perhaps, as in the linear case, this behaviour can be avoided by a gauge transformation which boosts our system in an appropriate way or by imposing some condition on the sources which eliminates this problem altogether.

\section{Conclusion}

We have presented the equations which arise as necessary conditions for the existence of a minimum for the total ADM energy of isolated systems along 
asymptotically flat space-like hypersurfaces, and given some arguments for the existence of solutions to such equations. While for the linear case we obtain a unique initial data set for each suitable source distribution, for the fully non-linear case we obtain, for each suitable source distribution as many initial data sets as flat backgrounds. These are physically different initial data sets corresponding to physically different final source distributions. This is not a new problem [12], and it arises from the fact that a source distribution does not acquire a physical character until a metric is given. From examples one sees that this non-uniqueness is actually needed for one to be able to account with this formalism for all physically interesting source distributions, and thus it is not necessary to further restrict the initial data sets to obtain uniqueness. For small enough sources we expect to find at least a relative minimum close to the flat data, in this case the final source configuration will not differ substantially from the one given a priori. For strong sources one would have to rely on one's own physical insight to guess the appropriate configuration to be given. Perhaps one could follow an approach similar to the one of Dixon [13] and try to define new variables by which invariant information about the matter content can be given.

It should be interesting to derive a similar procedure for the case where instead of considering initial data sets on a space-like hypersurface one considers null data in a light cone. One would then minimize the Bondi Energy, and obtain similar equations to those derived above. Since for the null case there is more geometrical structure a priori available one expects here to be able to fix the sources in a more geometrical way and therefore to obtain a smaller set of null data for each source distribution. Another advantage in this case is that one expects then our procedure to be roughly equivalent to the one imposing zero gravitational data at $j^{-}$.

Consider a hydrodynamical system consisting of two fluids, $A$ and $B$ say, whose individual sound speeds are very different, say $v_{A} \ll v_{B}$. If one is only interested in the long term evolution of fluid $A$, i.e. evolution on time scales of the order of (length of the system) $/ v_{A}$, and believe that the sound waves of fluid $B$ do not affect seriously such evolution, then the following numerical approximation is useful: At each time step use the full equations to determine component $A$, but use only the hydrostatic equations to determine component $B$. This approximation allows for longer time steps, and thus for a considerable saving in computer time, for the Courant-Friedrich-Lewy condition involves only component $A$. Are there systems gravitation-fluid where a similar approximation is appropriate? Since all propagation velocities are smaller or equal to that of the gravitational field this should be taken as component $B$. Do the minimal energy equations play the same role for these systems as the hydrostatic equations play for the two fluids? In general relativity there are no static equations unless very restrictive (equilibrium) conditions are imposed on the sources. The minimal energy equations represent the closest one can get to staticity without imposing any condition on the sources.

It should also be interesting to see the relation of this approach to those based on a Newtonian limit. Note that in our approach we do not require small velocities or stresses. The difference between these approaches can be seen already in electromagnetism. Consider as source for electromagnetism a charged rotating ring. The initial data obtained from the Newtonian limit have $\mathbf{E}=-\nabla \Phi$ such that $\Delta \Phi=-\varrho \mathbf{B}=0$, while the MEIDS has both $\mathbf{E}$ and $\mathbf{B}$ different from zero and just 
correspond to the stationary solution. From the minimal energy point of view the Newtonian limit initial data set corresponds to the MEIDS plus incoming radiation chosen in such a way as to make $\mathbf{B}=0$ in the hypersurface under consideration.

Acknowledgements. It is a pleasure to thank A. Ashtekar, D. Brill, J. Ehlers, A. Hemmerich, R. Penrose, B. Schmidt, and J. Winicour for useful ideas and criticisms.

\section{References}

1. Landau, L., Lifshitz, E.: The classical theory of fields. Reading, Pennsylvania, Addison-Wesley (1951)

Damour, T.: Phys. Rev. Lett. 51, 1019 (1983)

Futamase, T., Schutz, B.F.: Phys. Rev. D 32, 2557 (1985)

Isaacson, R.A., Welling, J.S., Winicour, J.: J. Math. Phys. 26, 2859 (1985)

Winicour, J.: J. Math. Phys. 24, 1139 (1984); 25, 2506 (1987); Gen. Ret. Grav. 19, 281 (1983)

Walker, M., Will, C.M.: Phys. Rev. Lett. 45, 1741 (1980)

Anderson, J.L.: Phys. Rev. Lett. 45, 1745 (1980)

2. Wald, R.M. (1984): General relativity, Chicago, IL: The University of Chicago Press. Appendix E (1984)

3. The spaces $H_{\sigma, \delta}$ are Weighted Sobolev Spaces, for a definition, description of their properties and applications see [4]. For most of the results in this paper it suffices to say that they are Hilbert spaces which code very well the differentiability and decay properties of their components. Thus, for example in $\mathbb{R}^{3}$, which is always to be our case, $H_{2,-1}\left(\mathbb{R}^{3}\right)$ contains all the continuous functions which decay as $1 / r$. When the elements of these spaces are 3 -vectors we denote them $H_{\sigma, \delta}$, when they are symmetric 2-tensors we denote them $H_{\sigma, \delta}^{*}$

4. Choquet-Bruhat, Y., Christodoulou, D.: Acta Mathematica, Vol. 146 (1981)

5. Moncrief, V.: J. Math. Phys. 16, 3, 493 (1975); 17, 10, 1893 (1976)

6. Arnowitt, R., Deser, S., Misner, C.W.: The dynamics of general relativity. Gravitation. Witten, L. (ed.). New York: Wiley

7. See for example [2, p. 80]

8. In fact, one can solve for this problem Eqs. $24 \mathrm{a}, \mathrm{b}), 25$ explicitly. Since the source has spherical symmetry, the solution $g_{a b}$ will also be spherically symmetric, and so, conformally flat. It is not difficult to see that the whole system of equations reduces (as far as the conformal factor, $\Omega$, is concerned) to the contraint equation. Choosing the radial function $r$ such that

$$
a_{r} \phi= \begin{cases}\frac{\phi_{0}}{L} & r<L \\ 0 & r>L .\end{cases}
$$

we obtain the following equation for $u=\ln \Omega$

$$
\partial_{r}^{2} u+\frac{2 \partial}{r} r u-\left(\partial_{r} u\right)^{2}=\frac{\phi_{0}^{2}}{4 L^{2}} \theta(L-r) .
$$

From the above equation it can be seen that the mass of the system depends linearly on $L$, and so we have obtained a one parameter family $(L)$ of solutions where the limiting on with $L=0$ has concentrated the source to a point and has zero mass. The tangent to this one parameter family of solutions must have a component along the gauge space as defined by Range $\left(\begin{array}{cc}0 & -1 / \sqrt{g} \\ 1 / \sqrt{g} & 0\end{array}\right) D \Phi^{*}$, which is not the physical gauge space since on it the sources are held fixed

9. Hawking, S.W., Ellis, G.F.R.: The Large Scale Structure of Space-Time. (Cambridge Monographs on Mathematical Physics), p. 229 (1973) 
10. Reula, O.: A configuration space for Quantum Gravity and solutions to the Euclidean Einstein equations in a slab region. Max-Planck-Institut für Astrophysik, Green report, MPA 275 (1987)

11. Cartan, H.: Differential Calculus. London: Kershaw Publishing

12. In fact, to solve the scalar constraint one also chooses a metric background and source configuration, $\varrho$, and then solves for the conformal factor

13. Dixon, W.G.: Extended bodies in general relativity: their description and motion. In: Isolated Gravitating Systems in General Relativity, p. 156

Ehlers, J. (ed.): Proc. of the Int. School of Physics Enrico Fermi. Course LXVII (1979) and Phil. Trans. Roy. Soc. Lond. 227, 59 (1974)

Communicated by S.-T. Yau

Received May 21, 1987; in revised form February 22, 1988 